Gdańsk 2019, Nr. 41

https://doi.org/10.26881/sgg.2019.41.01

Werner Abraham

Wien / München

\title{
Deutsche Modalpartikel in Nichthauptsatz- und Infinitkonstruktionen ${ }^{1}$
}

Ich zeichne die Leistungen und Vorkommensbedingungen der deutschen Modalpartikel anhand verschiedener Fragen vor, die immer schon im Mittelpunkt analytischer Beschreibungen gestanden haben (vgl. Whitт 2015), die aber heute deutlicher formuliert werden können, vor allem unter Bezug auf die Commongrounddimension, die zwischen Vorkontext und der aktuellen Äußerung bzw. Sprecher und Adressat vermittelt. Spezielle Aufmerksamkeit gilt den Beschränkungen, unter denen Modalpartikel in abhängigen Sätzen erscheinen.

Schlüsselwörter: anaphorisches Topik, Common ground, syntaktische Satzautonomie, Diskurs- und Dialogstruktur, Modalillokution, semantische Selbständigkeit, Topik-über ${ }^{2}$

Modal particles in dependent sentences and nonfinite phrases. - I survey the discourse effects and conditions of selection of the German modal (discourse) particles on the basis of different questions, that have always been in the focus of analytical descriptions (cf. WHITT 2015), which, however, can be formulated more clearly today. Special attention will be paid to the dimension of the Common ground that mediates between the prior context and the current utterance or speaker and addressee. Special focus is laid on the restrictions under which modal particles appear in dependent sentences.

Keywords: French, illocutionary adverbials, Japanese, modal particles (German), modal verbs (German), sentence and particles (Japanese), thetic constraints

1 Ich danke den Zuhörern bei der Konferenz Modalität und Aspektualität/Temporalität aus kontrastiver und typologischer Sicht an der Universität von Gdańsk am 28-29.09.2018 für wertvolle Diskussion. Ich danke ferner einem anonymen Gutachter für wertvolle Hinweise. - Die Mahnung, der Plural heiße Modalpartikeln, verfolgt mich mein ganzes wissenschaftliches Leben. Die eingemahnte schwache Pluralform entspricht aber nicht meinem Sprachgefühl - das, so meine ich, deutscher ist als das mancher anderer, eben auch dem der Dudenautoren.

2 Folgende Abkürzungen sind in Verwendung: $\mathrm{CG}=$ common ground (Diskursverständnisgemeinsamkeit zwischen Sprecher und Adressat), DMV = deontische Modalverblesart, DP = determiner phrase (Nomen + Artikelform), DP-Imperfektivität (Aspektentscheidung aufgrund von schwacher, d.h. indefiniter Nominalreferenz), DP-Perfektivität (Aspektentscheidung aufgrund von starker, d.h. definiter Nominalreferenz), EMV = epistemische Modalverblesart, FINKnoten $=$ syntaktische Position für finites Prädikat (eine der beiden Verbklammern je nachdem, ob Haupt- oder Nebensatz), Fok = Fokus, FORCE-Potential = modale Funktion eines Prädikats $($ Sprechakt), GA-Hauptsatz = japanischer Hauptsatz der kategorischen Zuweisung (Gegenteil von WA-Hauptsatz), liMF = linkes (Satz)Mittelfeld, MP = Modalpartikel (im Deutschen und Niederländische; Diskurspartikel im engeren Sinne), OV = Grundwortstellung Objekt vor Verb (Valenzlinksrektion im Deutschen und Japanischen); RS = Relativsatz, SE = Satzendpartikel (im Japanischen), Top $=$ Topik, VK = Verbklammer, VO = Grundwortstellung Verb vor Objekt (Valenzrechtsrektion wie im Englischen). 


\section{Einführung: Waltereits Klage}

„Muss man alle Diskurspartikel an den Eigenschaften des Deutschen messen?" (Richard WALTEREIT - SLE-Konferenz Tallinn 2018, WS on ,Discourse particles'). Man vergleiche französisch quand même unter Waltereits Anspruch, analytisch Ähnliches zu leisten wie deutsche modale Diskurspartikel/MP. Vergleichen wir.

(1) a Tun'imagines quand même pas que...

„Du stellst dir trotzdem nicht vor, dass ...”

$\mathrm{b} \quad$ jepeux quand même pas tout savoir

„Ich kann trotzdem/wie dem auch sei nicht alles wissen."

c C'est quand même pas sage que...

(2) a J'ai bien réfléchi.

„Das ist aber (doch) nicht klug, dass ...."

„Ich habe wohl nachgedacht."

b *J'ai réfléchi bien.

„Ich habe gut nachgedacht."

c *Jaibien réfléchi bien

vom Deutschen her intendiert: „Er hat wohl gut nachgedacht.“

So ist J'ai bien réfléchi bien (intendiert „Ich habe wohl gut drüber nachgedacht“) nicht korrekt, bien muss immer vor dem Partizip (wo wir im Deutschen das Mittelfeld ansiedeln) stehen. Eine Art Minimalpaar liegt in folgendem Satzpaar vor, wo Akzent (Großbuchstaben) die Bedeutungen trennt (P.-Y. Modicom, pers. Mitteilung am 22. Okt. 2018):

(3) Ce problčme est difficile: jyy ai bien /déjŕ réfléchi quelques JOURS\, mais rien n'y fait: Das Problem ist heikel. „Ich habe sehr wohl bereits mehrere TAGE darüber nachgedacht, aber es hilft nichts.“

(4) Ce problčme est difficile: j’y ai déjŕ /BIEN réfléchi quelques jours \, mais il va falloir que j'en fasse encore plus. „Das Problem ist heikel: Ich habe schon mehrere Tage lang GUT nachgedacht, aber ich muss noch mehr machen."

Was sind aber die Eigenschaften deutscher Modalpartikel, vornehmlich die das Deutsche eindeutig kennzeichnenden? Zu beachten ist jedenfalls, Diskurspartikel allein an Bedeutung zu messen scheint vermessen und unwissenschaftlich. Wir suchen nach formalen Anhaltspunkten.

Gehen wir von einem typologischen Hauptkennzeichen aus, das für deutsche und niederländische MP gilt: die OV-Bedingung. Diese gilt auch fürs Japanische, das über Satzendpartikel verfügt. Decken sich deren Funktionen mit denen des Deutschen?

(5) Japanische Satzendpartikel/SEP komme nur in Hauptsätzen (des ga- wie wa-Status) vor: ame-da-yo - es regnet doch

$$
\begin{array}{lll}
k a: & \text { Frage - } & \text { etwa } \\
n e: & \text { bestätigend }- & \text { ja } \\
y o: & \text { deklarativ }- & \text { eben } \\
z o: & \text { vergleichbar } y o, \text { nur gröber, selten aus dem Munde von Frauen } \\
z e: & \text { vergleichbar } z o \text { 'noch', nur gröber, nie aus dem Munde von Frauen }
\end{array}
$$




$\begin{array}{lll}\text { sa: } & \text { abschwächend, "glaube ich“ } & \text { aber } \\ \text { na: } & \text { in Betracht ziehend; vglbar ne,- } & \text { ja } \\ \text { ga: } & & \text { aber } \\ \text { no: } & \text { erklärend, fragend - } & \text { eben, ja } \\ \text { wa: } & \text { abschwächend, entrüstet; Frauensprache } \\ \text { kashira: } & \text { Frauensprache } & \text { vielleicht } \\ \text { yo: } & & \text { doch }\end{array}$

Zuerst zum syntaktischen Szenario. Nach TANAKa (2017) sind die japanischen Satzendpartikeln im Nebensatz ausgeschlossen. Tritt die Satzendpartikel in einer nebensatzverdächtigen Umgebung (z.B. einem Komplementsatz mit -to ,dass') auf, wird der betreffende Satz als direkte Wiedergabe, also als direkte Rede, verstanden.

(7) a Udo-wa kuru-to i-tta. Udo sagte, dass er kommt.“

Udo-wa kommen-dass sagen- PRÄT

b Udo-wa kuru-yo-to i-tta. Udo sagte: „Ich komme doch.“

Udo-wa kommen-SE-dass sagen-PRÄT

Im Japanischen ist die Grenze zwischen direkter und indirekter Rede ambig, aber vermittels Satzendpartikeln oder Höflichkeitsformeln lässt sich festmachen, dass der Satz direkte oder indirekte Wiedergabe einer Rede ist. Die Satzendpartikeln können aber auch in einem GA-Hauptsatz, also in thetischer Antidiskursfunktion auftreten.

(7) c Udo-ga kuru-yo. Udo kommt doch. bzw. ,Es kommt doch Udo.

Udo-GA kommen-SE

In diesem Fall wird nicht „Udo“, sondern der ganze Sachverhalt, also „Es kommt der doch, der Udo“ dem Hörer gegenüber bestätigt bzw. gefragt. Damit ergibt sich folgender Unterschied zwischen Diskurspartikeln im Deutschen und Japanischen: Die japanischen Sen sind in kategorischen (mit -wa) ebenso wie thetischen Sätzen (mit -ga) selegiert; im Deutschen dagegen sind MP in thetischen Sätzen ausgeschlossen.

Zur Semantik von (4). Der Vergleich zwischen Japanisch und Deutsch ergibt ein uneinheitliches Bild insofern, als die japanischen SEn neben attitudinaler Bedeutung auch eindeutig die Funktion von Honorifika erfüllen. Damit deckt sich ihre Funktion nicht mit der der MP. Wir ziehen den Schluss, dass OV (also Valenzlinksläufigkeit beim Satzaufbau) kein hinreichendes Kennzeichen der deutschen MP ist. Japanische Satzendpartikel haben nicht dieselben Eigenschaften wie die deutschen MPs, obwohl sie unter OV-strukturellen Bedingungen stehen. Adverbiale stehen im Deutschen in der rechten Hälfte des rechten Mittelfelds (Frey \& Pittner 1998), rechts von der strukturellen Position der deutschen MPs (ABRAHAM 1995/2005).

MPs sind so etwas wie Adverbiale, aber eben sehr spezifische: grammatische (Nullprojektionen), völlig unbeweglich (an das rechte links von den richtigen vollprojizierten Adverbialen im rechten Satzmittelfeld gebunden). Vgl. in (8), wo die deutschen MPs stehen müssen. 
(8) $\left[_{\mathrm{CP}}\right.$ Im Garten zweimal den Rasenmäher sorgfältig zerlegt $\left[_{\mathrm{liMF}} \mathrm{e}\left[_{\mathrm{C}^{\prime}}\right.\right.$ hat $\left[_{\mathrm{reMF}} j\right.$ a/aber/doch/eben meines Wissens ${ }^{*}[j a / a b e r / d o c h / e b e n]$ gestern ${ }^{*}[j a / a b e r /$ doch/eben $]$ ein Kollege ${ }^{*}[j a / a b e r / d o c h /$ eben] mit einem Schraubenzieher.

\begin{tabular}{|l|l|l|l|l|l|}
\hline VF & 1.VK & liMF & li-reMF & re-reMF & 2. VK \\
\hline $\begin{array}{l}\text { Im Garten zweimal den } \\
\begin{array}{l}\text { Rasenmäher } \text { sorgfältig }_{2} \\
\text { zerlegt }_{3}\end{array}\end{array}$ & hat & $\mathrm{e}_{1}$ & $\begin{array}{l}\text { ja/aber/ } \\
\text { doch/eben }\end{array}$ & $\begin{array}{l}\text { meines Wissens gestern ein Kol- } \\
\text { lege mit einem Schraubenzieher e }\end{array}$ & $\mathrm{e}_{3}$ \\
\hline
\end{tabular}

$\mathrm{e}_{1,2,3}=$ empty/leer nach Versetzung ins Satzvorfeld/VF

Die Frage ist natürlich, was eine OV-Satzstruktur im Gegensatz zu VO-Strukturen bietet, um MP-ähnliche Leistungen zu erbringen. Was ist mehr als OV nötig zur funktionalen Projektion von MPs? Verfügen andere Sprachen neben dem Deutschen über nichtanaphorisches, trotzdem topik-bezogenes MF? Wieso leistet gerade dieses die für MPs nötige syntaktische Struktur im Mittelfeld zwischen den beiden Verbklammern, Comp und Vletzt? Genauer wissen wir, dass zu den MP-Selektionsbeschränkungen die Anweisungen in (9a-g) gehören.

(9) MPs sind unversetzbar und selegierbar unter:

a Hauptsatzstatus (Rootstatus)

b sprechaktautonomer Nebensatzform (abhängiger Adverbialsatz, nichtfaktiver Ergänzungssatz, nichtrestriktiver/ appositiver Relativsatz)

c Attribut zu N ( \pm definit, unter jeder Quantelung)

d autonomer Infinitivphrase

e Wh-Adjunktion (einziges Mobilitätsziel um deutschen Satz)

f als konkreter Teil von (9c): dass/wh-Autonomie: Exklamativ/Kundgabe (d.h. Hauptsatzform $\neq$ Root sentence, vgl.: Dass er bloß kommt!)

MPs sind dagegen nicht selegierbar:

(10) a in Sätzen am Textanfang

b in Sätzen mit analytischem Erklärstatus wie Definitionen $\left(1+1={ }^{*}\right.$ aber 2$)$

c in Einleitungssätzen von Witzen und Märchen (mit präsentativer Funktion wie Es war einmal ein ...).

Wieso?

(11) Es scheint kein Zusammenhang zu bestehen zu Carlsons essentiellen Prädikaten (Individual level predicates/ILP) und Ereignisprädikationen (Stage level predicates/ SLP).

(10) bei Präsentativfunktion im sog. „Existenzsatz“ (Es steht ein Ungeheuer vor der Tür, Es gibt Bisons im Garten). Wieso?

(12) beim thetischem ( $\neq$ kategorischem) Urteil (d.h. bei Subjektinversion/VP-

Inkorporation, bei nichtnominativischen Subjekten wie in Mir geht es schlecht/Ihn schwindelt, bei unpersönlichen Passiven Es wird getanzt). Wieso?

Die folgenden Abschnitte gehen näher auf die Erscheinungen in (9a-g) und (10)-(12) ein. Ziel wird es sein herauszuschälen, welche Erscheinungen MP-Kandidaten anderer Sprachen mit dem Deutschen teilen und was zu diesen Erscheinungen an strukturellen Voraussetzungen gegeben sein muss. Zum Schluss kommen wir auf „Waltereits Klage“ zurück und ziehen 
Schlussfolgerungen. Davor gehen wir aber zu einem wichtigen Zwischenschritt zwischen den MP-Voraussetzungen in (9) und den funktionalen Bedingungen in (10): vgl. Abschnitt 2.

\section{Standortdifferenz zwischen epistemischen Modalverben und Modalpartikeln}

Sowohl bei epistemischen Modalverben/EMV als auch bei den deutschen Modalpartikeln geht es um epistemische Evaluierung von Propositionen, die Ereignisse bzw. Zustände beschreiben. Was jedoch ist dabei die Perspektive von EMV? Es ist eine intransitive Relation, weil die Standortbestimmung (Betrachterversetzung / engl. Displacement) in Sprecher und Betrachter als Evidenzquelle innerhalb einer einzigen Person (Sprecher $=$ Viewer $/$ Betrachter) lokalisiert sind. Man vgl. Das muss ein UFO sein (Sprecher = epistemischer Beurteiler der Erscheinung).

Dies steht ganz im Gegenteil zu MP: MPs bilden hinsichtlich der Evidenzquelle eine transitive Relation insofern, als sich Sprecher-Standpunkt (CG-Wissenshintergrund des Sprechers) und Adressaten-Standpunkt (CG-Wissenshintergrund des Hörers) nicht mehr in einer einzigen Person lokalisiert finden. Alleine die Tatsache, dass ein CG zwischen Sprecher und Adressaten aufgebaut wird, um den Diskurs auszuhandeln und zu einem Ergebnis zu bringen, setzt eine transitive epistemische Beziehung in Bezug auf $p$ ein. EMV- und MP-Epistemik sind verschieden: der Epistemikoperator für EMV ist einwertig, der für MP dagegen zweiwertig (AвRAHAm 2016a). Dies lässt sich im folgenden Abschnitt gut verfolgen.

\section{Modalpartikel in Haupt- u. Nebensätzen}

Modalpartikel wie ihre epistemischen Modalverbpartner sind in erster Linie an Satzfinitheit gebunden. Nur dann, so sagen wir, sind die wesentlichen Bedingungen für epistemische Satzbeurteilung (Assertivität, Wahrheitswert, Sprechaktstatus) gegeben. Nun gilt aber Satzsubordination als nichtassertiv (CRIstofaro 2003, 2008, 2013, 2014). Daraus würde folgen, dass MP in untergeordneten Sätzen ungrammatisch sind. Es zeigt sich jedoch gegen diese generelle Assertionsbedingung, dass bestimmte Nebensätze MPs zulassen.

(13) a Erkannjawoblschreiben

(14) b Er sagt, dass er ja wohl schreiben kann

c Er sagt, er kann ja wobl schreiben

(15) a Er leugnet, dass er *ja *wobl schreiben kann

... MP im Hauptsatz

... MP im Nebensatz1

...MP im (Brücken)Nebensatz2

b Er schaut zu, während/derweil sie *ja wobl/*eben schreibt ... *MP Nebensatz4

(16) a Erschaut zu, wogegen sie ja/eben schreibt $\quad .$. MP im Nebensatz5

b Dass sie mir das ja nicht/eben NICHT abschreibt! ... MP im Nebensatz6

c Eintritt ${ }^{*} j a /{ }^{*}$ eben $/{ }^{*}$ leider $/{ }^{O K}$ da $/{ }^{O K}$ polternd $/{ }^{O k}$ plötzlich Lady MacBeth.

... * MP im Hauptsatz7

Was macht MP in bestimmten Nebensätzen unmöglich? Konfigurieren wir die Nebensätze näher: 
(17) a Nebensatz $1=$ \#ergänzungssatz nach nichtfaktivem Performativerb $\quad$-> MP

b Nebensatz 2 = Ergänzungssatz ohne Subjunktion nach nichtfaktivem Performativerb

Nebensatz 3 = Ergänzungssatz nach faktivem Performativerb

d Nebensatz $4=$ temporaler Adverbialnebensatz

e Nebensatz $5=$ logisch-adversativer Adverbialnebensatz

$\rightarrow{ }^{*} \mathrm{MP}$

f Nebensatz $6=$ sprechaktautonome Nebensatzform

$\rightarrow{ }^{*} \mathrm{MP}$

g Hauptsatz $7=$ texteinleitender (thetischer) Hauptsatz

$\rightarrow \mathrm{MP}$

$-{ }^{*} \mathrm{MP}$

Die (Neben)Satztypen unter Kriterium der MP-Selektion laden zu folgenden grundsätzlichen Fragen ein. Vgl. (18)-(19).

(18) Was macht den Nebensatz 3 (nichtfaktives Brückenkomplement) anders als die anderen Satze? Der Brückensatz ist wie die direkte Rede nach dem Sagenverb (Performativverb), also wie ein Hauptsatz - MP-Selektion ist lizensiert, d.h. die illokutiven Operatorvoraussetzungen, die der MP-Einsatz voraussetzt, sind gegeben. Der Satz ist attitudinalautonom (dialogautonom).

(19) Was macht die Nebensätze 3+4 anders als Satz 7 unter dem MP-Selektionskriterium?

a Nebensatz 3 ist faktiv. D.h. das Hauptsatzprädikat, im vorliegenden Fall leugnen, setzt voraus, dass der Ergänzungsnebensatz assertiven Status bekommt. Was immer unter Leugnung stimmt, das Ereignis im Nebensatz ist geschehen. Das Brückenkomplement ist nicht möglich. Der Ergänzungsnebensatz ist modal-attitudinal nicht autonom, kann dementsprechend keine MP aufnehmen.

b Nebensatz 4 ist ein temporaler Adverbialnebensatz. Die Subjunktionen während/derweil schaffen Ereignisabhängigkeit des Nebensatzes vom Hauptsatz, damit ist der Nebensatz modal-attitudinal nicht autonom, erlaubt also keine MP-Selektion.

c Nebensatz 5 ist in Gegensatz zu Nebensatz 4 ein logisch-adversativer Adverbialsatz. Es ist davon auszugehen, dass derartige logische Satzverknüpfungen (mit den Subjunktionen wogegen, weil etc.) ihre eigene Attitudinalautonomie haben, während die Ereignisverknüpfungen die des Hauptsatzes gemein haben. Daraus folgt, dass logische Nebensätze eigene Attitudinalautonomie haben, die ereignisverknüpfenden Nebensätze dagegen nicht.

Wir schaffen uns noch einmal einen Überblick über jene Satzformen und -funktionen, die MP-Autonomie zeigen.

\section{MP in speziellen Nebensätzen}

MPs lassen sich in manchen Nebensätzen selegieren, in anderen nicht.

(20) Bei Ereignisverknüpfung ${ }^{*} \mathrm{MP}$ :

a Er schrieb, als sie ${ }^{*} j a /{ }^{*}$ eben kochte

b Nur jene Männer, die *ja Voyeure sind,

... * temporaler Adv-Satz

... * restriktiver Relativsatz

MP in Logische Fügung OKMP:

(21) a ER/ ${ }^{*}$ Er schrieb, wogegen $\mathrm{SIE} /{ }^{*}$ sie ${ }^{*}$ ja $/{ }^{*}$ eben kochte

... logischer Adv-Satz

b Männer, die ${ }^{O K}$ ja alle Voyeure sind,

... appositiver Relativsatz 
MP und Fokusakzent: weil+V2!

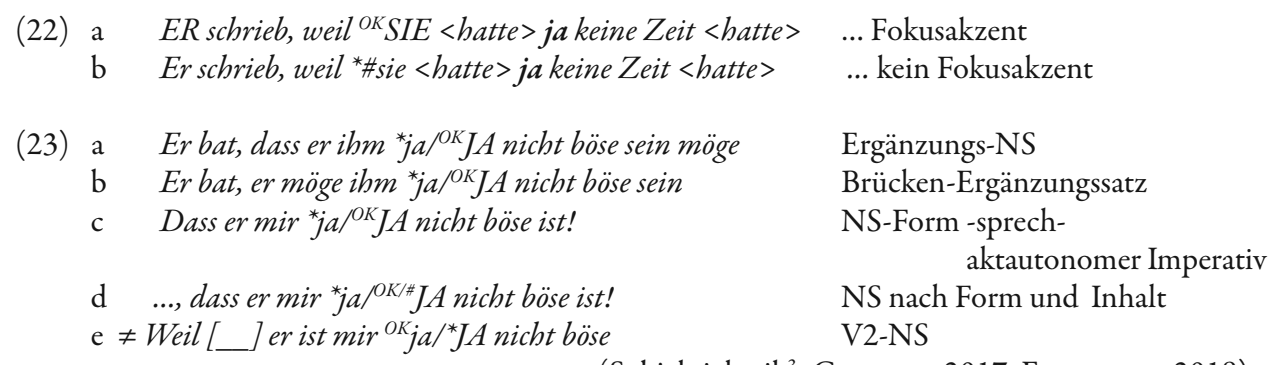

(Subjektivlogik ${ }^{3}$ : CATASSO 2017, FREYWALD 2018)

Das MP-Selektionskriterium eröffnet also ein ganz neues Autonomieszenario. Unter syntaktisch-semantischem Gesichtspunkt können Nebensätze Präsuppositionsstatus (nichtfaktive Verben) und Assertivstatus (faktive Verben) haben. Hier wird nach ,Struktur-in-der-Struktur' geurteilt. Da MP jedoch Sätzen attitudinalen und diskurseinbettenden Status verleihen, kommt dem Unterschied von Nebensatzform und Nebensatzinhalt neue Bedeutung zu. (23c) hat Nebensatzstatus, völlig unabhängig davon allerdings Sprechakt- und damit inhaltliche Autonomie. Und darauf kommt es bei der MP-Selektion an: Modalpartikel brauchen inhaltliche Autonomie und lassen sich in dieser Eigenschaft als Lizensierer von inhaltlicher (Sprechakt-) Autonomie einsetzen. Modalpartikel fordern Satzillokutionspotential - und damit eine CG-Basis. Wir belegen dies alles - Syntaxstatus gegen Illokutionsstatus - noch einmal anhand faktiver Prädikation in (24).

(24) Faktizität eines transitiven Prädikats bedeutet, dass der illokutionäre Operator auf den Ergänzungssatz übertragen wird. D.h., dass das faktive Prädikat nicht nur eine Performanzkomponente enthält, sondern auch eine Sprechaktkomponente: $X P$ leugnet, dass $Y P=X P$ assertiert mit Leugnungskraft, dass YP. Der Ergänzungssatz unter dem Performanzverb leugnet ist also assertiert. Im Gegensatz dazu gilt: dass YP als Ergänzung zu XP sagt ist nicht assertiert, sondern hat Präsuppositionsstatus. ${ }^{4}$

3 Der Begriff der „Subjektivlogik“(АвRAнам 2016a, b, c) gründet auf der Beobachtung, dass Kausalsätze mit weil+V2, also mit Verbzweitstellung (statt der üblichen Nebensatzabfolge mit Vletzt) den kausalen Zusammenhang nicht unter Berücksichtigung der zu verknüpfenden Ereignisse, sondern auf rein subjektiver Grundlage herstellen. D.h. konkret, dass (i) Sie freut sich, weil er früh nachbause kommt eine andere Intensionalität ausdrückt als (ii) Sie freut sich, weil er kommt früh nachhause. Zu (i) passt die Frage, ,Wann freut sie sich?', zu (ii) dagegen, Was sagt sie, wenn sie sich freut?'. V2 unter der Subjunktion weil entfaltet also so wie die Kausalkoordination denn eine subjektive Begründung, eine klar weniger ereignismotivierenden Zusammenhang etwa in dem Sinne Sie freut sich, weil (so meint sie) er kommt früh nachhause. Die Begründungsperspektiven sind verschieden und erlauben dem Betrachter unterschiedliche Schlüsse. Weder bei FreYwald (2018) noch bei Catasso (2017) allerdings wird dieser Perspektivenunterschied entfaltet und in eine erklärende Analyse eingebracht.

Cristofaros Feststellung "Hence subordination can be defined as a situation where a clause encodes non-asserted information. Any clause encoding this type of information can be regarded as an instance of subordination regardless of its formal properties." (CRISTOFARO 2016: 8) muss also in Bezug auf Ergänzungssätze unter faktiven Prädikaten entscheidend korrigiert werden. Überhaupt sind die Generalisierungen zum Präsuppositionsstatus von Satzunterordnung unter Bezug auf (20)-(23) neu zu formulieren. Festzuhalten ist, dass Sprechaktautonomie viele der syntaktischen und semantischen Einteilungskriterien von Nebensätzen (bei 
Unter dem MP-Selektionskriterium freilich eröffnet sich eine ganz andere Autonomierelation: MP-Selektion unter Faktivität ist ausgeschlossen, unter Nichtfaktivität dagegen lizensiert. Faktizität hebt die Generalisierung auf, dass Ergänzungsnebensätze präsupponiert und damit nicht assertiert sind. MP-Einsatzbarkeit hebt die Generalisierung auf, dass nur Hauptsätze Sprechaktstatus haben und damit der Satzform größeres Gewicht zuteilen als pragmatischinhaltlichem Gehalt. Die reine Ergänzungsnebensatzform kann ebenso wie adverbiale Nebensatzform Sprechaktautonomie erreichen und damit den MP-Einsatz legitimieren. Vgl. (23c, e) sowie (25a, b).

(25) a $\left[{ }_{\mathrm{CP} 1} \ldots\left[_{\mathrm{CP} 2}\right.\right.$ Wie $\left[\mathrm{MF}\right.$ der aber $\left[{ }_{\mathrm{VP}}\left[{ }_{\mathrm{V}}\right.\right.$ schwindelt $\left.\left.\left.\left.]\right]\right]\right]\right]$

Man vergleiche die folgenden Testformen. Präsupponiertes ändert seinen Nichtassertivstatus nach Negation des Matrixprädikats nicht.

(26) Faktiv: bereuen (bedauern, leugnen u.a.).

a Otto bereut, dass er *ja alles Bier ausgetrunken hat. (Folgerung: Er hat es austrunken ist assertiert)

b Otto bereut nicht, dass er *ja alles Bier ausgetrunken hat. (dieselbe Folgerung)

In beiden Fällen ist die Selektion von MP uninterpretierbar.

Nichtfaktiv: beweisen u.a.

c Kopernikus hat bewiesen, dass die Erde kugelformigja ist.

(Folgerung: Es ist eine gesicherte Tatsache, ist assertiert), dass die Erde kugelförmig ist).

d Kopernikus hat nicht bewiesen, dass die Erde ja kugelformig ist.

(die obige Folgerung entfällt, der Ergänzungssatz ist nicht assertiert, sondern bloß präsupponiert).

In beiden Fällen, (26c) wie (26d) ist die Selektion von MP idiomatisch gut interpretierbar.

Das MP-Selektionskriterium hat demnach einen ganz anderen Hintergrund als die Unterscheidung zwischen assertierbaren und nicht assertierbaren (präsupponierten) Nebensätzen. Assertierbarkeit hängt mit dem FORCE-Potential des FIN-Knotens (Wahrheitswertbeurteilung) zusammen, das Gelingen der MP-Selektion ist unabhängig von FIN durch Sprecherdeixis (Common ground), Sprechaktstatus und Modalität in FORCE bestimmt.

\section{Prosodie und Satzthetik als MP-Selektoren}

Einfluss auf MP-Nichteinsetzbarkeit haben thetische (im Unterschied zu kategorischen) Sätzen. Sie wiederum unterliegen eigenen Prosodiegesetzlichkeiten.

\footnotetext{
Hutprosodie (angezeigt durch steigende und fallende Prosodie, //... II)

//

(27) Richtig AUF regt mich ${ }^{\text {oK }}$ ja/*vielleicht GAR NICHTS
}

Cristofaro, aber auch anderen wie Comrie (2008) und LANGacker (2008), um nur wenige zu nennen) entscheidend überschreibt. Vgl. AвRAнам (2016a,b,c sowie 2019). 
Der letzte Satz, (27), hat zwei Interpretationen: eine mit Kontrastakzent $A U F$ im Vorfeld (etwa im Kontrast zu $A B($ regt); und eine präsentativ-thetische Interpretation mit Hutprosodie, die ein Satzganzes schafft wie ausgedrückt in $\left(27^{\circ}\right)$.

(27’) $\left[_{\mathrm{CPES}}\right.$ regt $\left[\left[_{\text {liMF }}\right.\right.$ mich [re $\mathrm{re}_{\mathrm{MF}}\left[\mathrm{VP}_{\mathrm{VP}}\right.$ GAR NICHTS auf $\left.\left.]\right]\right]$...Präsentativthetik mit Normalprosodie

Thetische Sätze sind diskursunabhängige bzw. diskurseinleitende Sätze. Solche finden sich im Japanischen grundsätzlich mit der Kasuspartikel -ga (im Unterschied zu -wa) gekennzeichnet. Das Deutsche hat solche morphologische Mittel zur Identifikation von Thetik nicht, setzt aber vor allem Präsentativsätze mit Subjektinversion in diskurseinleitender Funktion ein ( $\left[_{\mathrm{CP}}\right.$ Es war einmal [ ${ }_{V P}$ ein König ...]]). Auch Hutprosodie lässt sich wie (27) als Thetikidentifikator einsetzen, dann allerdings schließen sie kontrastive Lesarten aus, da sie diskursuneingebunden (eben 'thetisch') sind. Die Verbpartikel $A U F$ - im Vorfeld, der Normalposition des Subjekts, ergibt jedoch rhematisierende Versetzung des Subjekts ins Mittelfeld/MF, wodurch mehr Spannung (mit Behaghel 'Erregung') entsteht.

An Satzthetik verbindet sich auch die Setzung von Verumfokus. Vgl. (29) mit (28). //

11

(28) Richtig AUF regt mich ${ }^{{ }^{\circ}}$ JAMP/*vielleichtMP NICHTS ...Deklarativ mit Hutprosodie

(29) Das IST $\mathbf{j a}_{\mathrm{MP}} /$ vielleicht $_{\mathrm{MP}}$ aufregend! ... Verumfokusexklamativ

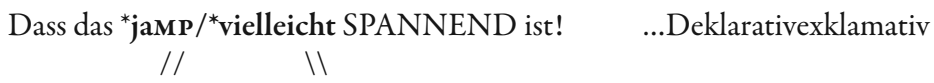

(31) Dass das *bloß/*ja//BLOß/JA SPANNEND wird! ...Aufforderungsexklamativ

(32) $\quad \neq(31)$ Das ist ja VIELLEICHT ${ }_{\text {ADV }}$ AUFREGENDDEFAULTAKZENTZ/*aufregend. ...nichtthetischer Deklarativ

Wir notieren vor allem die Akzentunterschiede bei den Modalpartikelschreibungen. Dies weist darauf hin, dass bei Akzentsetzung grundlegend unterschiedliche Lizenzen und Sprechaktvariationen vorliegen. Zudem zeigen sich die individuellen MP-Bedingungen: etwa zwischen der MP vielleicht und $j a$. Verumfokussetzung und Hutprosodie machen eigene Voraussetzungen für MP-Selektion (27) und (31). Es zeigt sich, dass MP im deklarativen NS ausgeschlossen sind (30), idiomatisch dagegen in exklamativem Aufforderungssprechakt (31).

Identifikatoren von Satzthetik sind also kurz zusammengefasst: Sätze mit leerem Vorfeld/SpezCP und Subjektinversion (VP-internem Subjekt), Sätze mit Hutprosodie, Sätze mit Verumfokus und, wie wir noch sehen werden, Sätze mit betontem Subjekt im Vorfeld. Exklamative scheinen grundsätzlich in thetischer Verwendung zu sein. Allen diesen Formen allerdings lassen sich auch nichtthetische Interpretationen zuordnen. Es gibt im Deutschen keine eindeutige Form, die bloß und eindeutig Satzthetik identifiziert. 


\section{MP-Immobilität}

MPs lassen sich nicht verschieben. Ihr Platz im rechten Mittelfeld des deutschen Satzes links von allen Adverbialen, also noch außerhalb von VP, ist fest (mit einer einzigen Ausnahme, auf die wir noch zurückkommen). Zu dieser Versetzungsbeschränkung gibt es ein interessantes kategorienübergreifendes Pendant, nämlich Aspekt. Bei mereologischer Referenzkennzeichnung (mittels Ereignismerkmalen) zeichnet sich Merkmalskongruenz zu aspektueller Imperfektivität bzw. Perfektivität ab. Referenzmerkmale für DP lassen sich in innere [+teilbar] und äußere [-teilbar] einteilen. Dies ist genau die Scheidung von imperfektivem und perfektivem Aspekt. Es entspricht demnach aspektuelle Imperfektivität referentieller Determination in alle Münzen sind aus Gold, Perfektivität dagegen referentieller Einschränkung in nur Münzen, die aus Gold sind. Vgl. dazu (33) gegen (34) mit entsprechenden Foki.

(33) Die/Solche ja/doch goldenen MÜNZEN = = Münzen, die ja/doch golden sind $\quad$... nr/appositiver RS

(34) Die/Solche *ja/*doch GOLDENEN Münzen $=$... DP-Perfektivität = (JENE) Münzen, die ${ }^{*} j a /{ }^{*}$ doch golden sind $\quad$... restriktiver RS

(33) gilt für Münzen schlechthin, da sie alle golden sind. (34) dagegen gilt nur für Münzen, die aus Gold sind. MP-Selektion ergibt sich nur bei DP-Imperfektivität, scil. appositiver Relativsatzgrundlage, sie schließt sich bei DP-Perfektivität und restriktiver Relativsatzgrundlage aus. Diese Trennung reicht jedoch nicht aus, um Grammatisches von Ungrammatischem zu trennen. Nichtrestriktives (appositives) Attribut bzw. der zugrundeliegende Relativsatz müssen von maximierender Gradierung/Gr sein, um MP-Selektion möglich zu machen. Vgl. (35a-c).
a Sie trägt ihre Schuhe, die ja umwerfenden sind.
$\ldots$ nrRS $+\mathrm{Gr}$
b Sie trägt ihre Schuhe, die ja völlig abgetragen sind.
... nrRS+Gr
c Sie trägt ihre Schuhe, die *ja braun sind.
... nrRS-Gr

Die vergleichsweise ungradierte (unkomparierbare) Farbe braun lässt keine MP-Selektion zu, wie (36c) im Vergleich mit (36a, b) nochmals darstellt. ${ }^{5}$

Formale Attributrepräsentationen:

(36) a $*_{D_{D P}}$ ihre $\left.\left[{ }_{C P}\left[{ }_{A P} j a\right]_{i} C^{o}\left[{ }_{I P}\left[{ }_{D P} S c h u h e\right] \ldots{ }_{-} t_{i}\right]\right]\right] \quad \ldots$ ohne Einschränkung

$\mathrm{b}\left[{ }_{D P}\right.$ ihre $\left[{ }_{C P}\left[\mathrm{ja}\left[{ }_{G} \text { umwerfenden }\right]\right]_{\mathrm{i}} \mathrm{C}^{\circ}\left[{ }_{\mathrm{IP}}\left[{ }_{\mathrm{DP}}\right.\right.\right.$ Schuhe $\left.\left.\left.] \ldots \mathrm{t}_{\mathrm{i}}\right]\right]\right] \quad$...nrRS+GR

c $\left[{ }_{D P}\right.$ ihre $\left[{ }_{C P}\left[{ }_{A P}\left[{ }_{G}\right.\right.\right.$ ja völlig abgetragenen $\left.]\right] \mathrm{i} \mathrm{C}^{o}\left[{ }_{I P}\left[{ }_{D P}\right.\right.$ Schuhe $\left.\left.\left.] \ldots t_{i}\right]\right]\right] \ldots$ nur für rRS $+\mathbf{G r}$

Die einzige MP-Verschiebung aus dem Satzmittelfeld ergibt sich für ein $W$-Wort im Satzvorfeld. Diese MP-Adjunktion an $W$ ergibt als Abweichung von der MP-Normalposition oft mirative Wirkungen.

5 Es ist natürlich durchaus vorstellbar, dass es zu braun Vergleichswerte gibt, also bräuner bzw. weniger braun. Man denke an die ideologisch-politische Bedeutung von braun. 
(37) a Was LACHT er denn so?

b WAS denn / Was=n LACHT er so! (Unverständnis anzeigend)

(38) a Was macht das (denn) schon/bloß AUS?

b WAS schon/bloß macht das (denn) AUS! (ablehnend, da unverständlich)

Ich gehe davon aus, dass der Mirativeffekt gerade der Adjunktion der MP an FORCE im W-Wort geschuldet ist. Die modale Komponente der MPs ist generell durch die Sprecherdeixis in FORCE getragen (AbRAham 2016a, b, c; 2019). Ähnlich anderen Fällen von Voranstellung (,Fronting') ergibt sich ein modaler Sondereffekt.

\section{MP in infiniten Konstruktionen: Satzgrammatik oder Sprechakt?}

MPs treten in Infinitiv- und Partizipialphrasen auf, wie (39a, b. c) (vgl. GäRTNER 2017) zeigen, also bei Bechs erster und zweiter Verbalstufe, nicht jedoch bei der dritten. Vgl. (39b). $\left[{ }^{\# \#}\right.$ bedeutet sehr eingeschränkte Verwendung, da im vorliegenden Fall der Anweisung auf offiziellen Schipisten unverständlich]

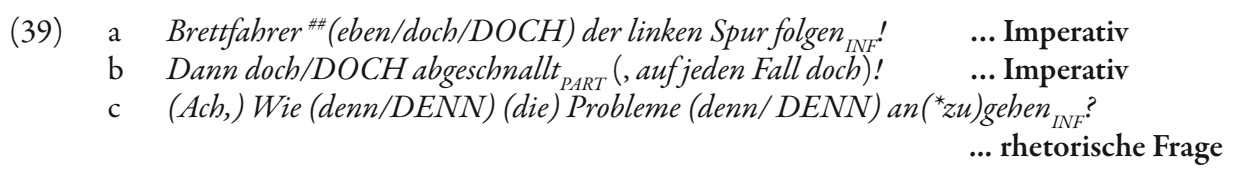

Infinitivphrasen sind keine CP-Konstruktionen, da der Tempusknoten (und damit der Zusammenhang mit FORCE zur Wahrheitsbewertbarkeit) unbenützt bleibt, trotzdem erlauben Infinitivphrasen MP-Selektionen, dies allerdings nicht als Satzoperatoren, sondern als Sprechaktoperatoren. Zugrunde liegt der Umstand, dass bloße Infinitive und zweite Partizipien in Imperativ- und Fragefunktion verwendet werden können.

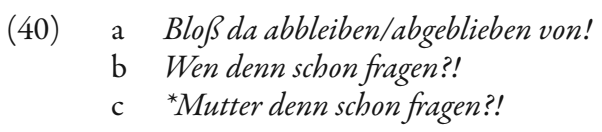

$$
\begin{aligned}
& \text {... Imperativ } \\
& \text {... rhetorische } w \text {-Frage } \\
& \text {... Entscheidungsfrage }
\end{aligned}
$$

Auszugehen ist davon, dass diese Sprechaktautonomie in Unabhängigkeit vom Satz- und Finitheitsstatus mit der Idiomatik von (40a, b) im Gesprächsdeutsch einhergeht: Man kann, entsprechende Kontexte vorausgesetzt, mit Infinitiven und zweiten Partizipien Kommandierbzw. Fragehandlungen durchführen.

\section{MP und Szeneneinstellung}

Da MPs diskurs- bzw. dialogeingebunden sein müssen und entsprechend spezifische CGs schaffen, sind sie in thetischen (text- und gesprächseinleitenden) Sätzen nicht einsetzbar. 
(41) a Es war *eben $/{ }^{*}$ ja einmal ein König zu Thule.

b Trat da *eben/*ja ein stämmiger Matrose in die Hafenkneipe.

... Sageneinleitung

c Trafen sich *eben $/{ }^{*}$ ja Hase und Igel am Ackerrand.

d Willst Du mir (denn WIRKLICH) helfen?

In (41d) weist der Einsatz von denn WIRKLICH darauf hin, dass eine Gesprächsfortsetzung vorliegt, ein CG bereits geschaffen ist, der weiterverhandelt werden will. Ohne diesen Einzug liegt eine Gesprächseinleitung vor, in der die MPs eben und ja unpassend sind. Siehe die strukturelle Voraussetzung für MP-Selektion in (42).

$$
\left[_ { \text { Forcep } } \mathrm { MP } _ { \text { mod } } \cdots \left[_ { \text { Topp } } \mathrm { MP } _ { \text { dial } } \cdots \left[_ { \text { Focp } } \cdots \left[_ { \text { Topp } } \mathrm { MP } _ { \text { TopAbour/CG } } \cdots \left[_{\text {Finp }} \cdots\right.\right.\right.\right.\right.
$$

Bei Thetik, die ja keine MP-Selektion erlaubt, liegt mit der Beschneidung der diskursorientierten Strukturknoten Topik und Fokus implizit auch der Verlust von Topik-about/TopAbout vor (vgl. Haegeman 2010). Diese Strukturbeschneidung ist radikal insofern, als auch der Illokutionsknoten in FORCE verloren geht, von dessen Existenz MP-Selektion abhängt.

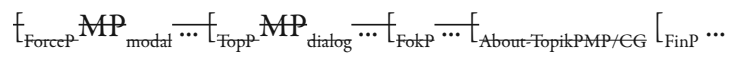

Die Haegemanhypothese wird also hier erweitert: Das Fehlen von FORCEP impliziert Fehlen von äußerem TopP und innerem/About-TopP ebenso wie FokP. Damit erfolgt die Trennung von Diskurs-/Dialogeinbindung, von der Voraussetzung zur MP-Selektion.

Was bedeutet Subjektinversion in diesem Zusammenhang? Thetische Sätze, darunter solche mit Subjektinversion, erlauben ja kein MP. Was ist Thetik, was macht Subjektinversion in der Funktion der Präsentativsyntax? Wiederum sprechen wir Haegemans Implikationshypothese an: Mit der thematischen Nullbesetzung des Satzvorfelds sind nicht nur alle diskurs- und dialogeinbindenden Operatoren gestrichen, sondern auch der Illokutionsoperator in FORCE. Fehlende Vorfeldbesetzung (wie in Sätzen mit expletivem $E s$ in der Subjektposition) raubt dem deutschen Satz nicht nur Diskursstatus, sondern auch das Modalitätspotential für MP-Selektion.

Szeneneinstellungen im Sinne von (44a, b) verhindern demnach MP-Selektion insoferne nicht, als sie das gesamte diskurseinbindende Kategorieninventar (nach Rizzis CP-Expansion) aufrechterhalten. Nur wenn das Satzvorfeld als Diskursindikator thematisch unbesetzt ist, ist der Diskurs zum Satz abgeschnitten. Es entsteht der thetische Satz, der MPs ausschließt.

(44) a. $H T / M P>$ Scene Setter $\mathbf{M P}>L D / M P>F I N>V P \quad$ (angelehnt an BENINCÁ \&

Poletto 2004, Haegeman 2012, Cognola 2013)

b. Frame $/ \mathbf{M P}>[$ About-Top $/ \mathbf{M P}>$ Kontrast-Top $/ \mathbf{M P} /$ Fok $>$ Familiar-Top $/ \mathbf{M P}$ $>F I N>V P$

(FrasCARELLI \& HINTERHÖLZL 2007)

Mobilität der MP ist schon deshalb aufgehoben, weil sich die relative Ordnung der MPs nach folgender Skopushierarchie in (45) ergibt. Diese relative Ordnung bestimmt sich nach den Quellkategorien, aus denen sich die MP durch Grammatikalisierung entwickelt haben. Man beachte, dass MPs grammatische Kategorien, also Nullprojektoren sind (АвRAHAм 2019).

\footnotetext{
6 "The highest area of the left periphery hosts the frame, where FPs for hanging topics (from here on HT)
} and scene setters are located." (BENINCÀ/PoletTo 2004: 78) 
(45) C1-INTERJECTION > C2-COORD > C3-ADV > C4-FP > C5-CONTR

$[\mathrm{C}=$ Nullprojektion $=$ grammatische Projektion (es gibt kein SpezMP) $]$

$\mathrm{C} 1$

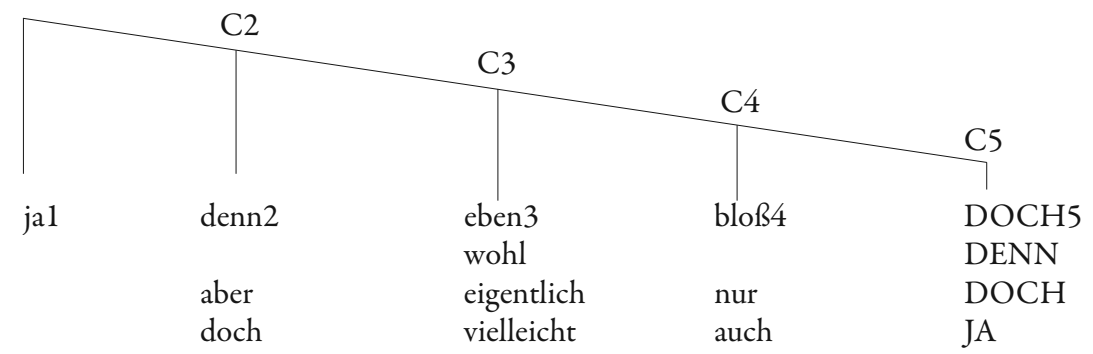

(46) a Wie denn2 bloß4 soll ich leben?

b *Wie bloß4 denn2 soll ich leben?

Das Niederländische, eine der wenigen V2/OV-Sprachen, schließt sich bei der relativen MPOrdnung dem Deutschen an.

(47) a $\left[_{C}\right.$ dat $\left[_{\text {limF }}\right.$ of ik het hem $\left[_{\text {reMF }}\right.$ dan toch weer eens $\left[_{v / \mathrm{VP}}\left[_{\mathrm{ADV}}\right.\right.$ een keer $\left[_{\mathrm{V}^{\prime}}\right.$ uitgelegd heb]]]]]]

„dass ob ich es ihm dann doch wieder einmal noch einmal erklärt habe“

b *dat ik het dan toch weer eens ${ }^{*}\left[_{\mathrm{liMF}}\right.$ hem een keer uitgelegd heb.

c dat ik dan toch weer eens V $_{\mathrm{VP}}$ een boek heb gelezen.

„dass ich dann doch wieder einmal ein Buch gelesen habe."

d $\quad$ dat ik * [ ${ }_{\mathrm{VP}}$ een boek dan toch weer eens gelezen heb]

e Doe dat dan nu toch maar weer eens even $\left[_{\mathrm{VP}}\right.$ over.

„Mach das dann nun doch nur wieder einmal gerade noch einmal.“

\section{Schlussfolgerungen - Gemeinsamkeiten}

Wir schreiten zu ersten Schlussfolgerungen.

(48) Syntax: MPs besetzen eine eigene Position, die strukturelle Topik-about-Position im Satzmittelfeld. Es scheint sich diese Position in der Geschichte des Deutschen ausgebildet und gefestigt zu haben (АвRAнам 1990, 1991; van Gelderen 1992). Typologisch mag dies bedeuten, dass eine Sprache ohne Topik about-Strukturstelle keine MP (der deutschen, engen Definition) projizieren kann. Vgl. (49).

(49) [CP Co [liMF Anaphern/Definita [reMF [About-Topik MP [VP...V]]]

Diese syntaltische Repräsentation teilen alle Konstruktionen mit Subjektinversion/VP-Inkorporation, darunter Konstruktionen mit nichtnominativischen Subjekten wie in Mir geht es schlecht-Es geht mir schlecht/Ihn schwindelt-Es schwindelt ihn und mit unpersönlichen Passiven Es wird (von allen) getanzt. Vgl. AвRAH Aм \& Leiss (2006).

(50) About-Topik impliziert die Existenz eines Commn ground/CG. MPs sind nicht nur diskursbzw. dialogeingebunden, sondern sie sind auch CG-Manipulatoren. D.h. MPs schaffen Dialogeinbettung mit Sprecher-Adressaten- Aushandlung des CG (wie bei Der ist ja blöd, was auf Zustimmung durch den Adressaten hinweist). 
(51) MPs lizensieren sich letztlich durch eigenen Sprechaktstatus, worauf MP-Selektion in nichtfiniten Konstruktionen und in Attributen zu DPs hinweist (motiviert durch Attributrelativsätze).

(52) MPs verfügen über Potential für Mirativausdrücke (in Kooperation mit der Adjunktion an $W$-Ausdrücke im Satzvorfeld, bei Abweichung von Satznormalakzent, bei Verumfokus, bei Passung von semantisch scheinbar unverträglicher Koordination (ABRAHAM 2017).

(53) MP-Kombinationen weisen kategorienmotivierte Reihenbildung nach ihrer lexikalischen Quelle auf, aus der sich die MPs durch Grammatikalisierung gebildet haben und neu bilden.

(54) Wir haben festgestellt, dass MP-Selektion im nichtrestriktiven (appositiven), nicht jedoch im restriktiven Relativsatz möglich ist. Daraus folgt natürlich nicht, dass nur der nichtrestriktive Relativsatz Dialogeinbettung erlaubt. Vgl. 'Innere DP-Referenz (mit MP-Selektion) $\neq$ äußerer DP-Referenz (ohne diese Selektion, d.h. ${ }^{*} \mathrm{MP}$ ). Trotzdem: Was erklärt, dass innere DP-Referenz mit MP-Selektion zu tun hat?

(55) Wir halten fest, dass sich bei $W$-Adjunktion der sonst völlig immobilen MP spezielle mirative Wirkungen einstellen.

(a) WAS =n/ denn LACHT er so! (Unverständnis)

(b) WAS schon/bloß macht das (denn) AUS! (Ablehnung)

Wir haben daraus den Schluss gezogen, dass die MP bei dieser SpezCP-Adjunktion in den Strukturbereich der Sprechaktfunktion und der Illokution (Attitudinalität) in FORCE gerät. Dies bestätigt den besonderen illokutionären Status des Topik-About-Strukturknotens.

Es stellt sich angesichts dieser aus den Daten herausgelesenen Zusammenhänge folgende Hauptaporie ein: Wenn Dialog-/ Diskurseinbettung das Hauptkriterium für MP-Selektion ist, wie passt dann das Vorkommen von MP bei appositiven (nicht jedoch restriktiven) Relativsätzen und bei logischen (nicht jedoch ereignisbasierten) Nebensätzen unter dieses Kriterium? Was haben nreRS und logNS mit Diskurseinbettung zu tun? Wie jedoch wenn MP-Selektion bloß CG-Aufrollung voraussetzt, dass gerade die Existenz von CG im Sinne von (42)-(43) alle anderen Voraussetzungen wie Diskurs- und Dialogeinbindung, Illokution und damit FORCE mitvoraussetzt?!

\section{Diskurspartikel oder Modalpartikel?}

Haben Diskurspartikel in anderen Sprachen als dem Deutschen und Niederländischen nicht die Diskurs- und Dialogqualität der deutschen MP deshalb, weil sie den MP-Vorgabekriterien nicht entsprechen? In erinnere an „Waltereits Klage“: „Muss man alle Diskurspartikel an den Eigenschaften des Deutschen messen" (Richard Waltereit bei seinem SLE-Vortrag in Tallin 2018 über französische Diskurspartikel wie in (57)).

(56) a Tun'imagines quand même pas que „wie dem auch sei/trotzdem nicht ...“

b Jepeux quand même pas tout savoir "trotzdem nicht"

c Il est bien réfléchi

(i) „Es ist wohl verstanden“ oder (ii) „Es ist wohlverstanden“

D.h. handelt es sich um ein MP-Pendant (i) oder das Adv. zu réfléchi (ii)? 
Wir halten auf jeden Fall fest: Diskurspartikel im engeren (CG-aushandelnden) Sinne allein an lexikalischer Bedeutung zu messen wäre vermessen und würde dem Problem, d.h. dem Vergleich, nicht gerecht werden. Man vergleiche die Forderung von Harald WeydT (1989: 338):

„Die Frage, um die es hier wirklich geht, ist vielmehr: Wie würde ein alter Mann, der im Deutschen so spricht wie Borcherts alter Mann, in analoger Situation auf Französisch sprechen?' Am besten würde der Übersetzer also ganz vergessen, daß es im Deutschen Partikeln und dieser Sprache spezifische Ausdrucksmittel gibt. Er sollte sich statt dessen bemühen, die durch den deutschen Text erfaßte Intention [...], gemäß den Mitteln, die die französische Sprache bereitstellt, in einer Weise teilzuerfassen, daß ein französischer Leser einen Gesamteindruck erschließen kann, der dem eines deutschen Lesers möglichst nahe kommt.“

(57) i Sprecher: Je me demande souvent, est-ce que jécris „comme même“ ou bien "quand même"?

ii Adressat:_Ich spreche nicht Französisch.

iii Sprecher: C'est quand même.

iv „Das ist (ja eben) gleich/dasselbe/wurst."

Ou bien in (57i) entspricht zweifellos dem deutschen oder wobl, aber ebenso einem oder ebenso oder vielleicht auch. iv meint wohl, was in iii gesagt wird, d.h. es würde wohl am besten der Weydtschen Forderung entsprechen - aber es steht nun einmal nicht da. In (58) habe ich in das Zitat aus Faust die Strukturklammern eingefügt; quand même ist klar ein Hauptsatzkonnektor, keine MP.

(58) $\left[_{\mathrm{CP1}}\right.$ Les pauvres gens ne soupçonnent jamais le diable, $\left[_{\mathrm{CONN}}\right.$ quand même $\left[_{\mathrm{CP} 2}\right.$ il les tiendrait $\rightarrow$ la gorge]]. (Johann Wolfgang von Goethe Faust)“

\begin{tabular}{|l|l|l|l|l|l|l|}
\hline CP1-Vorfeld & VP & CONN & CP2 & MF & {$\left[_{V P}\right.$ V } & OBJ-ADV $]$ \\
\hline $\begin{array}{l}\text { Les pauvres } \\
\text { gens } \\
\text { Die Armen }\end{array}$ & $\begin{array}{l}\text { ne soupçon-nent le } \\
\text { diable } \\
\text { beargwöhnen den } \\
\text { Teufel nicht }\end{array}$ & quand même & il & les & tiendrait & à la gorge \\
sie & $\begin{array}{l}\text { wird } \\
\text { kriegen }\end{array}$ & $\begin{array}{l}\text { an der } \\
\text { Gurgel }\end{array}$ \\
\hline
\end{tabular}

Die wörtliche Übersetzung von (58) gliedert sich wie in (59).

(59) $]_{\text {CP-VORfeld }}$ Dennoch/Und doch/Trotzdem/Nevertheless $\left[_{C^{\prime}}\right.$ wird $\left[_{\text {limF }}\right.$ er sie $\left[_{\text {reMF }}\left(? d\right.\right.$ o ch / $\left.{ }^{?} \mathrm{ja}\right)$ $\left[_{\mathrm{VP}}\right.$ an der Gurgel kriegen

\begin{tabular}{|l|l|l|l|l|l|}
\hline$\left[_{\mathrm{CP}}\right.$ Vorfeld & Verbklammer & liMF-Anaphern & reMF-MP & reMF-Adv & Verbklammer \\
\hline Dennoch & wird & er sie & $($ 'ja/?doch $)$ & an der Gurgel & kriegen \\
\hline
\end{tabular}

(60) $\left[_{\text {CP-VORfeld }} \operatorname{Er}\left[{ }_{C^{c}}\right.\right.$ wird $\left[_{\text {limF }}\right.$ sie $\left[_{\text {remF }}\right.$ trotzdem $/{ }^{*}$ doch $/{ }^{*}$ ja $/ \mathbf{D O C H}$ an der Gurgel $[\mathrm{VP}$ kriegen]]]]]

\begin{tabular}{|c|c|c|c|c|c|}
\hline$\left[{ }_{C P}\right.$ Vorfeld & Verbklammer & liMF-Anaphern & reMF-MP & reMF-Adv & Verbklammer \\
\hline Er & wird & Sie & $\begin{array}{l}\text { trotzdem } /{ }^{*} \mathrm{ja} / \\
{ }^{*} \text { doch } / \mathrm{DOCH}\end{array}$ & an der Gurgel & kriegen \\
\hline
\end{tabular}


In (58)-(61) zeigt sich anhand von Bedeutungsäquivalenten ebenso wie den Strukturanalysen, dass quand même keine Diskurpartikel im engeren Sinne (MP) ist.

(61) a Pour vous en souvenir vous pouvez vous rappeler que: "Quand même" est similaire à "Quand bien même"

b "[ $\left[_{\mathrm{CP} 1}\right.$ Quand bien même [ ${ }_{\mathrm{CP} 2}$ il pleuvrait $\left.]\right],\left[_{\mathrm{CP} 3}\right.$ je serais present $\left.\left.]\right]\right]$."

c "Tu m’a blessé mais $\left[_{\mathrm{CP}}\right.$ je $\left[\mathrm{VP}\right.$ t'aime $\left[_{\mathrm{MF}}\right.$ quand même $\left.\left.]\right]\right]$ ]" $\neq$ SYNTAKTISCH $/ \cong$ SEMANTISCH

$\left[\left[_{\mathrm{CP}} \text { Quand bien même tu m'a blessé, }\right]_{\mathrm{i}} \mathrm{je}\right.$ t'ai aimé $\left.\mathrm{t}_{\mathrm{i}}\right]$

Wir halten fest, dass die MP-Epistemik einen Perspektivenwechsel insofern enthält, als der Adressat auf die Spr-Einladung hin den CG-Inhalt nach einer Spr-fremden Perspektive weiter verhandeln möchte. D.h. es gelten für MPs die folgenden Vorgaben:

(62) a subjektive Propositionsgeltung, indem Sprecherhaltung vorliegt und

b Interaktion zwischen Sprecher und Adressaten

c Perspektivenwechsel, d.h. Versetzung (displacement):

Spr versetzt von sich selbst, d.h. Spr=Betrachter

Spr versetzt von Adr, Spr $\neq($ Betrachter $=$ Adressat $)$

Der folgende Abschnitt führt den Unterschied auf eine noch deutlichere Analyseebene.

\section{MP als CG-Operatoren}

Die Gesprächsgrundlage (Common ground/CG, auch Question under discussion (Qud) oder deutsch Quaestio) setzt Diskurs- und Dialogkonnex voraus. Sie bringt in den Komplex der Überlegung zwischen Sprecher und Adressanten ein, was bei den beiden Kommunikationspartnern vorausgesetzt wird und schrittweise (CG1>CG2>...) den Diskurs bzw. Dialog voranbringt (dabei positives Aushandeln ebenso nachzeichnend wie Scheitern eines Aushandelns bzw. Festhalten an und Zurückkommen zu früheren CG-Schritten).

(63) DOCH wird er sie ...] $]_{A P O D O S I S}: D O C H$ präsupponiert (füllt CG mit) Implikation (konversationeller Implikatur), dass sich Voraussetzung und $p$ widersprechen, d.h. $\Rightarrow \Leftarrow$.

$\mathrm{CG}(\mathrm{CP} 1=$ nicht beargwöhnen:: $\rightarrow$ er wird ihnen nichts Böses tun $) \Rightarrow \Leftarrow[\mathrm{CP} 2 \mathrm{er}$

kriegt sie an der Gurgel)

Wobei: $\Rightarrow \Leftarrow$ für Hauptsatzkonnexion

Zu lesen als: Aus dem Vorkontext, CP1 (sie beargwöhnen den Teufel nicht) ist erwartbar, dass er ihnen nichts Böses tun wird (CP2). Daraus wiederum folgt über die Verknüpfung mit $D O C H$, dass er ihnen Böses tun wird (sie an der Gurgel kriegen wird).

(64) Er wird sie doch nicht an der Gurgel kriegen] $]_{A P O D O S I S}$ : doch präsupponiert (füllt CG mit) Implikation, dass kein Gegensatz zu $p$ (ØрÜ).

$\mathrm{CG}(\mathrm{CP} 1=$ nicht beargwöhnen $:: \rightarrow$ er wird ihnen nichts Böses tun $) \Rightarrow(\neg \Rightarrow \Leftarrow)[\mathrm{CP} 2 \mathrm{er} \mathrm{kriegt}$ sie an der Gurgel)

$\mathrm{Zu}$ lesen als: Aus dem Vorkontext, CP1 (sie beargwöhnen den Teufel nicht) ist erwartbar, dass er ihnen nichts Böses tun wird (CP2). Daraus wiederum folgt über die Verknüpfung mit doch, 
dass der Widerspruch ihn nicht davon abhält, dass er ihnen Böses tun wird (sie an der Gurgel kriegen wird).

(65) Er wird sie ja nicht ...:ja präsupponiert (füllt CG mit) Implikation, dass $\neg(\Rightarrow \neg)$.

$\mathrm{CG}(\mathrm{CP} 1=$ nicht beargwöhnen $: \rightarrow$ er wird ihnen nichts Böses tun $) \Rightarrow(\Rightarrow \neg)[\mathrm{CP} 2$ er kriegt sie an der Gurgel)

Zu lesen als: Aus dem Vorkontext, CP1 (sie beargwöhnen den Teufel nicht) ist erwartbar, dass er ihnen nichts Böses tun wird (CP2). Die Verknüpfung mit ja bestätigt die Erwartung, dass er ihnen nichts Böses tun wird (sie nicht an der Gurgel kriegen wird).

BRINTON (1996: 6) setzt in ihrer Untersuchung der mittelenglischen "pragmatic markers" gan, anon, gelamp, bifel, hwat, and I gesse, alle so etwas wie „meine ich“, die folgenden Unterscheidungsfunktionen auf Textebene an:

(66) a to mark various kinds of boundaries (to initiate or end a discourse or to effect a shift in topic);

b to assist in turn-taking in oral discourse or 'chunking' (marking of episode or paragraph) in written discourse;

und auf interpersonaler Ebene:

c subjectively, to express speaker attitude;

$\mathrm{d}$ interactively, to achieve between speaker and addressee (appealing to addressee or expressing shared or common knowledge).

(66d) entspricht dem Begriff des Common ground zur p-Verhandlung (bei Brinton unerwähnt).

\section{CG- und Sprechaktvorgaben für die individuellen MP}

CG- und Sprechaktvorgaben sind für jede MP unterschiedlich. Rhetorische Effekte gehen auf formale Abweichungen zurück, etwa die Spannung zwischen Satztypus und Sprechakt.

(67) a Du willst doch kommen!? CG: Sprecher-Zweifel, Sprecher-Erwartung, Sprecher-Warnung,

b Du willst ja auch kommen. CG: Sprecher-Bestätigungseinladung an Adressaten,

c Er will eben auch kommen. CG: Sprecher-Nachbegründung, Sprechervorgabe an Adressaten im Dialogkontext

$\mathrm{d} \operatorname{Der~IST}_{\mathrm{MP}} / *^{*}$ ist $_{\mathrm{ADV}}$ vielleicht ${ }_{\mathrm{MP}} /{ }_{\mathrm{ADV}}$ ein Gauner!

Als Adverb muss VIELLEICHT stark betont werden. Als MP (als funktionaler Nullprojektion) kann es nie betont sein. MP kann keinen Verumfokus tragen.

\section{About-Topik}

Was leistet die About-Topik-Strukturstelle/im reMF?

(68) a Dialog/Diskurs/Präkontext ( $\neq$ anaphorisch)

b Sprecherdeixis (in FORCE/modaler/CG-Operator 
Die Strukturposition „Topik-about-“ im deutschen Satzmittelfeld öffnet CG. Dies setzt ein Kategorienfeld zwischen V2 und Vletzt voraus. Wir haben zur Typologie des MPs geschlossen: „Wo kein About-Topik, dort auch keine MP im engeren, CG-struktur- und illokutionsschaffenden Sinne". Wir zeigen dies an der Variation von Definitheit und Wortstellung im Mittelfeld (anhand von Textstellen bei W. Borchert):

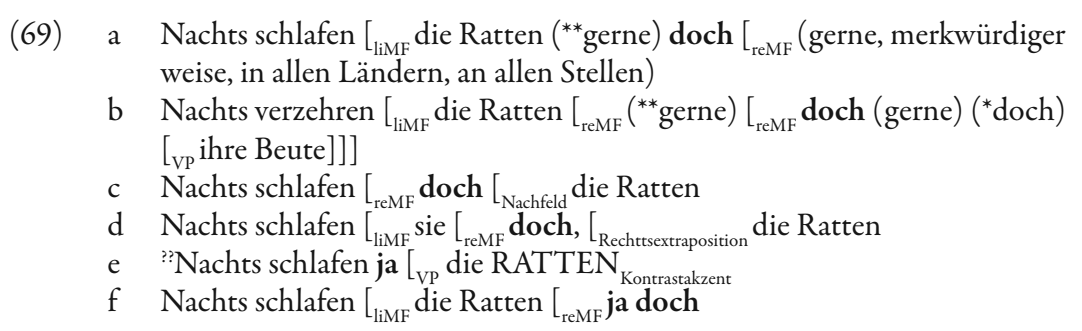

Das Satzfeld des Deutschen ist streng nach Position und Definitheit eingeteilt. Wo Versetzung nach Textbedingungen vorliegt, wird dies durch Kontrastakzent angezeigt. Mit Ausnahme der Satzakzentdefaultposition am tiefsten Kopf der VP gelten andere Akzente als aus ihren Grundpositionen verschobene Argument- und Adverbialpositionen. Das valenztechnisch definierte Subjekt im Satzvorfeld (SpezCP) trägt im Defaultfall als einzige Konstituente keinen Akzent. D.h. alle Phrasen außer dem externen Argument/Subjekt, die im Satzvorfeld erscheinen, tragen Kontrastakzent.

\section{Schluss: Sprechen Sie eine Epistemik- oder eine MP-Sprache?}

Es gilt seit ABRAHAM \& LEISS (2014) für MV intransitive Modalität.

(70) a Er muss MV $_{\text {gerne in Danzig sein }}$ Imperfektiv $\quad .$. EMV

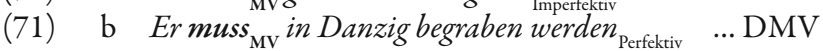

Es gilt MV+Perfektiv (muss sterben) = DMV, während MV+Imperfektiv (muss in Danzig sein $)=$ EMV. Der Relationsbegriff modaler Intransitivität geht darauf zurück, dass Sprecher und Betrachter eins sind - daher ,innere ('intransitive' - Sprecher=Viewer) Perspektive', Spr $=$ Adr.

Im Gegensatz dazu gilt für MP transitive Epistemizität.

(72) Er muss ja auch/eben auch/schon auch $]_{\text {pr } \neq \text { Adr }}$ in Danzig begraben werden.

Sprecher verhandelt über CG mit dem Adressaten/Betrachter außerhalb von sich: Spr $\neq$ Adressat - es gilt äußere ('transitive' Sprecher $\neq$ Betrachter/Viewer) Perspektive. D.h. die Modalitätskategorien MV und MP unterscheiden sich nach Stellenwertigkeit: MV(Spr/ Adr $) \neq \mathrm{MP}$ (Spr, Adr). Nur die Wertigkeit (Spr, Adr) impliziert CG-Status. Der Sprecher macht mit MP dem Hörer/Adressaten ein Verhandlungsangebot. Die einzelnen MP-Lexeme signalisieren jeweils unterschiedliche Spr-Hypothesen zum nächsten CG-Stand $(j a \neq$ eben F schon). Zu (Spr/Adr) gibt es keinen CG.

Es lässt sich zu Waltereits Klage die Frage halten: Hat Ihre, des Lesers Sprache eine sprecher-hörer-deiktische Intransitiv- oder Transitivgrammatik? Ihre individuelle Antwort ist 
auch die Antwort auf die Frage, ob Ihre Sprache Diskurspartikel der engen (MP) oder der weiteren Analytik besitzt. Freilich: Die weitaus interessantere Frage ist, was hinter einem solchen typologischen Unterschied steckt. Ob die hier vorgelegte Antwort das letzte Wort ist?

\section{Bibliographie}

Aвraham, Werner 1990. Zur heterogenen Entfaltung der Modalpartikel im Ahd. und Mhd. In: A. BetT EN \& C.M. RieHL (Hg.): Neuere Forschungen zur historischen Syntax des Deutschen, 124-138. Tübingen: Niemeyer.

Abraham, Werner 1991. The grammaticalization of the German modal particles. In: E. Clooss TraUGOTT \& B. HeINE (eds.): Grammaticalization. Vol. 2: 331-380. Amsterdam: John Benjamins.

Aвraham, Werner 32013 . Deutsche Syntax im Sprachenvergleich. Tübingen: Stauffenburg.

ABraham, Werner 2016a. Die deutschen Entsprechungen zu omdat und want: Nur weil und denn? In: Sander Lestrade, Peter De Swart \& Lotte Hogeweg (eds.): Addenda. Artikelen voor Ad Foolen, 1-12. Radboud Universiteit Nijmegen. (2015.

Aвraham, Werner 2016b. Was bedeutet Subordination mit V2 im Deutschen und Niederländischen: omdat und want ebenso wie weil und denn? In: Leuvense Bijdragen 99-100 (2016): 122-132 [Sprache in Raum und Geschichte, System und Kultur. Festschrift für Luk Draaye. Redigiert von K. Feyaerts, G. Brône, S. Schoonjans \& G. Stuyckens].

AвRAHAM, Werner 2016c. Zum grundsätzlichen Unterschied von Ereignissubjunktion (de re) und Prämissensubjunktion (de dicto) im Westgermanischen. In: Glottotheory 7(2): 113-136.

DOI 10.1515/glot-2016-0011z

Abraham, Werner 2017. Modalpartikel und Mirativeffekte. In: S. TANAKa, E. Leiss, W. Abraham \& Y. Fujinawa (Hg.): Grammatische Funktionen aus Sicht der japanischen und deutschen Germanistik, 76-108 [Linguistische Berichte Sonderheft 24]. Hamburg: H. Buske.

Abraham, Werner 2018. Non-at-issue phenomena in German, Dutch, and Japanese: exclamatives and thetics. Paper abstract for Clause typing and the syntax-to-discourse relation in head-final languages: Konferenz Konstanz May 15-17, 2018.

Aвraham, Werner 2019. Modality. Cambridge: CUP (Im Erscheinen).

ABRAHAM, Werner \& Elisabeth LeIss 2006. Personal and impersonal passives: Definite vs. indefinite diatheses. In: TRANSACTIONS OF THE PHILOLOGICAL SOCIETY 104: 230-259.

ABraham, Werner \& Elisabeth Leiss 2014. Introduction. In: E. Leiss \& W. Abraham (eds.): Modes of modality. Modality, typology, and universal grammar, 1-15 [Studies in Language Complementary Series 149]. Amsterdam: John Benjamins.

Averina, Anna 2015. Partikeln im komplexen Satz. Frankfurt: P. Lang.

Benincá, Paola \& Cecilia Poletto 2004. Topic, Focus and V2: defining the CP sublayers. In: The Structure of CP and IP. The Cartography of Syntactic Structures, 52-75. Vol. 2, Luigi RizzI (ed.). Oxford: Oxford University Press.

BlüHDORN, Hardarik 2013. Besprechung von Marco Coniglio 2011. Die Syntax der deutschen Modalpartikeln. Ibre Distribution und Lizenzierung in Haupt-und Nebensätzen [(Studia grammatica 73]. Berlin: Akademie Verlag. In: Zeitschrift für Dialektologie und Linguistik LXXX/ (2013): 206-209. 
BRINTON, Laurel J. 1996. Pragmatic markers in English. Grammaticalization and discourse markers. Berlin: De Gruyter.

BRÜNJES, Lena (2014). Das Paradigma deutscher Modalpartikeln: Dialoggrammatische Funktion und paradigmeninterne Oppositionen [Reihe Germanistische Linguistik, Band 301]. Berlin: De Gruyter.

Comrie, Bernard (2008). Subordination, Coordination: Form, Semantics, Pragmatics. In E.J. VAIdA (Hg.): Subordination and Coordination in North Asian Languages, 1-16. Amsterdam and Philadelphia: John Benjamins.

Coniglio, Marco 2011. Die Syntax der deutschen Modalpartikeln. Ihre Distribution und Lizenzierung in Haupt-und Nebensätzen [studia grammatica 73]. Berlin: Akademie Verlag.

Coniglio, Federica 2013. Syntactic Variation and Verb Second: A German Dialect in Northern Italy [Linguistik Aktuell 201]. Amsterdam: John Benjamins.

Cristofaro, Sonja (2003). Subordination. Oxford: Oxford University Press.

CRISTOFARO, Sonja (2008). Asymmetric events, subordination, and grammatical categories. In B. LEWANDowsKa-Tomaszczy (ed.): Asymmetric events, 151-172. Amsterdam: John Benjamins.

CRISTOFARO, Sonja (2014). Is subordination a grammatical category of particular languages? In J.K. Ilona Herlin \& L. VISAPÄÄ (ed.): Contexts of subordination - cognitive, typological and discourse perspectives. Amsterdam: John Benjamins.

CRISTOFARO, Sonja 2016. Subordination in cross-linguistic perspective (1) Some traditional assumptions about subordination (Vortragsvorlage basierend aufCRISTOFARO 2003: chap. 2; 2014): 1-14.

Frascarelli, Mara \& Roland Hinterhölzl 2007. Types of topics in German and Italian. In: Kerstin Schwabe \& Susanne Winkler (eds.): On information structure, meaning and form, 87-116. Amsterdam: John Benjamins.

Frey, Werner \& Karin Pittner 1998. Zur Positionierung der Adverbiale im deutschen Mittelfeld. In: Linguistische Berichte 176: 489-534.

GÄRTNER, Hans Martin 2017. Root infinitivals and modal particles. An interim report. In: J. BAYER \& V. Struckmeier (eds.): Discourse particles, 115-143. Berlin: De Gruyter.

Gelderen, Elly van 1992. Tense and to in Layamon. In: Folia Linguistica Historica 13/1-2 (1992/3): $133-142$

Haegeman, Liliane 2012. Adverbial clauses, main clause phenomena, and composition of the left periphery. Oxford: OUP.

LANGACKER, Ronald W. (2008). Subordination in cognitive grammar. In: B. LEWANDowsKATomaszczy K (ed.): Asymmetric events, 137-49. Amsterdam: John Benjamins

TANAKA, Shin 2017. Suche nach latenter Invarianz bei genetisch fremden Sprachen am Beispiel Deutsch-Japanisch. In: S. Tanaka, E. Leiss, W. Abraham \& Y. Fujinawa (Hg.): Grammatische Funktionen aus Sicht der japanischen und deutschen Germanistik, 59-74 [Linguistische Berichte Sonderheft 24]. Hamburg: H. Buske.

Weydt, Harald 1989. Partikelfunktionen und Gestalterkennen. In: H. WEYdT (Hg.): Sprechen mit Partikeln. Berlin: De Gruyter.

Whitt, Richard J. 2015. Besprechung von: Lena BRÜnJes Das Paradigma deutscher Modalpartikeln: Dialoggrammatische Funktion und paradigmeninterne Oppositionen. Berlin: De Gruyter 2014. In: Journal of Pragmatics 76: 181-183.

Zyватоv, Lew 1990. Was die Partikeln bedeuten. Eine kontrastive Analyse Russisch-Deutsch [Slavistische Beiträge]. München: Otto Sagner. 